\title{
A Note on the Symmetric Powers of the Standard Representation of $S_{n}$
}

\author{
David Savitt ${ }^{1}$ \\ Department of Mathematics, Harvard University \\ Cambridge, MA 02138, USA \\ dsavitt@math.harvard.edu \\ Richard P. Stanley ${ }^{2}$ \\ Department of Mathematics, Massachusetts Institute of Technology \\ Cambridge, MA 02139, USA \\ rstan@math.mit.edu
}

Submitted: January 7, 2000; Accepted: February 12, 2000

\begin{abstract}
In this paper, we prove that the dimension of the space spanned by the characters of the symmetric powers of the standard $n$-dimensional representation of $S_{n}$ is asymptotic to $n^{2} / 2$. This is proved by using generating functions to obtain formulas for upper and lower bounds, both asymptotic to $n^{2} / 2$, for this dimension. In particular, for $n \geq 7$, these characters do not span the full space of class functions on $S_{n}$.
\end{abstract}

Primary AMS subject classification: 05E10. Secondary: 05A15, 05A16, 05E05.

\section{Notation}

Let $P(n)$ denote the number of (unordered) partitions of $n$ into positive integers, and let $\phi$ denote the Euler totient function. Let $V$ be the standard $n$-dimensional representation of $S_{n}$, so that $V=\mathbb{C} e_{1} \oplus \cdots \oplus \mathbb{C} e_{n}$ with $\sigma\left(e_{i}\right)=e_{\sigma i}$ for $\sigma \in S_{n}$. Let $S^{N} V$ denote the $N^{\text {th }}$ symmetric power of $V$, and let $\chi_{N}: S_{n} \rightarrow \mathbb{Z}$ denote its character. Finally, let $D(n)$ denote the dimension of the space of class functions on $S_{n}$ spanned by all the $\chi_{N}, N \geq 0$.

\footnotetext{
${ }^{1}$ Supported by an NSERC PGS-B fellowship

${ }^{2}$ Partially supported by NSF grant DMS-9500714
} 


\section{Preliminaries}

Our aim in this paper is to investigate the numbers $D(n)$. It is a fundamental problem of invariant theory to decompose the character of the symmetric powers of an irreducible representation of a finite group (or more generally a reductive group). A special case with a nice theory is the reflection representation of a finite Coxeter group. This is essentially what we are looking at. (The defining representation of $S_{n}$ consists of the direct sum of the reflection representation and the trivial representation. This trivial summand has no significant effect on the theory.) In this context it seems natural to ask: what is the dimension of the space spanned by the symmetric powers? Moreover, decomposing the symmetric powers of the character of an irreducible representation of $S_{n}$ is an example of the operation of inner plethysm [1, Exer. 7.74], so we are also obtaining some new information related to this operation.

We begin with:

Lemma 1.1. Let $\lambda=\left(\lambda_{1}, \ldots, \lambda_{k}\right)$ be a partition of $n$ (which we denote by $\lambda \vdash n$ ), and suppose $\sigma \in S_{n}$ is a $\lambda$-cycle. Then $\chi_{N}(\sigma)$ is equal to the number of solutions $\left(x_{1}, \ldots, x_{k}\right)$ in nonnegative integers to the equation $\lambda_{1} x_{1}+\cdots+\lambda_{k} x_{k}=N$.

Proof. Suppose without loss of generality that $\sigma=\left(\begin{array}{llll}1 & 2 & \cdots & \lambda_{1}\end{array}\right)\left(\lambda_{1}+1 \cdots \lambda_{1}+\right.$ $\left.\lambda_{2}\right) \cdots\left(\lambda_{1}+\cdots+\lambda_{k-1}+1 \cdots n\right)$. Consider a basis vector $e_{1}^{\otimes c_{1}} \otimes \cdots \otimes e_{n}^{\otimes c_{n}}$ of $S^{N} V$, so that $c_{1}+\cdots+c_{n}=N$ with each $c_{i} \geq 0$. This vector is fixed by $\sigma$ if and only if $c_{1}=\cdots=c_{\lambda_{1}}, c_{\lambda_{1}+1}=\cdots=c_{\lambda_{1}+\lambda_{2}}$ and so on. Since $\chi_{N}(\sigma)$ equals the number of basis vectors fixed by $\sigma$, the lemma follows.

It seems difficult to work directly with the $\chi_{N}$ 's; fortunately, it is not too hard to restate the problem in more concrete terms. Given a partition $\lambda=\left(\lambda_{1}, \ldots, \lambda_{k}\right)$ of $n$, define

$$
f_{\lambda}(q)=\frac{1}{\left(1-q^{\lambda_{1}}\right) \cdots\left(1-q^{\lambda_{k}}\right)} .
$$

Next, define $F_{n} \subset \mathbb{C}[[q]]$ to be the complex vector space spanned by all of these $f_{\lambda}(q)$ 's. We have:

Proposition 1.2. $\operatorname{dim} F_{n}=D(n)$.

Proof. Consider the table of the characters $\chi_{N}$; we are interested in the dimension of the row-span of this table. Since the dimension of the row-span of a matrix is equal to the dimension of its column-span, we can equally well study the dimension of the space spanned by the columns of the table. By the preceeding lemma, the $N^{\text {th }}$ entry of the column corresponding to the $\lambda$-cycles is equal to the number of nonnegative integer solutions to the equation $\lambda_{1} x_{1}+\cdots+\lambda_{k} x_{k}=N$. Consequently, one easily verifies that $f_{\lambda}(q)$ is the generating function for the entries of the column corresponding to the $\lambda$-cycles. The dimension of the column-span of our table is therefore equal to $\operatorname{dim} F_{n}$, and the proposition is proved. 


\section{Upper Bounds on $D(n)$}

Our basic strategy for computing upper bounds for $\operatorname{dim} F_{n}$ is to write all of the generating functions $f_{\lambda}(q)$ as rational functions over a common denominator; then the dimension of their span is bounded above by 1 plus the degree of their numerators. For example, one can see without much difficulty that $(1-q)\left(1-q^{2}\right) \cdots\left(1-q^{n}\right)$ is the least common multiple of the denominators of the $f_{\lambda}(q)$ 's. Putting all of the $f_{\lambda}(q)$ 's over this common denominator, their numerators then have degree $n(n+1) / 2-n$, which proves

$$
D(n) \leq \frac{n(n-1)}{2}+1
$$

By modifying this strategy carefully, it is possible to find a somewhat better bound. Observe that the denominator of each of our $f_{\lambda}$ 's is (up to sign change) a product of cyclotomic polynomials. In fact, the power of the $j^{\text {th }}$ cyclotomic polynomial $\Phi_{j}(q)$ dividing the denominator of $f_{\lambda}(q)$ is precisely equal to the number of $\lambda_{i}$ 's which are divisible by $j$. It follows that $\Phi_{j}(q)$ divides the denominator of $f_{\lambda}(q)$ at most $\left\lfloor\frac{n}{j}\right\rfloor$ times, and the partitions $\lambda$ for which this upper bound is achieved are precisely the $P\left(n-j\left\lfloor\frac{n}{j}\right\rfloor\right)$ partitions of $n$ which contain $\left\lfloor\frac{n}{j}\right\rfloor$ copies of $j$. Let $S_{j}$ be the collection of $f_{\lambda}$ 's corresponding to these $P\left(n-j\left\lfloor\frac{n}{j}\right\rfloor\right)$ partitions. One sees immediately that the dimension of the space spanned by the functions in $S_{j}$ is just $D\left(n-j\left\lfloor\frac{n}{j}\right\rfloor\right)$ : in fact, the functions in this space are exactly $1 /\left(1-q^{j}\right)^{\left\lfloor\frac{n}{j}\right\rfloor}$ times the functions in $F_{n-j\left\lfloor\frac{n}{j}\right\rfloor}$.

Now the power of $\Phi_{j}(q)$ in the least common multiple of the denominators of all of the $f_{\lambda}(q)$ 's excluding those in $S_{j}$ is only $\left\lfloor\frac{n}{j}\right\rfloor-1$, so the degree of this common denominator is only $n(n+1) / 2-\phi(j)$. Therefore, as in the first paragraph of this section, the dimension of the space spanned by all of the $f_{\lambda}$ 's except those in $S_{j}$ is at most $n(n-1) / 2+1-\phi(j)$; since the dimension spanned by the functions in $S_{j}$ is $D\left(n-j\left\lfloor\frac{n}{j}\right\rfloor\right)$, we have proved the upper bound

$$
D(n) \leq \frac{n(n-1)}{2}+1-\phi(j)+D\left(n-j\left\lfloor\frac{n}{j}\right\rfloor\right)
$$

If it happens that $D\left(n-j\left|\frac{n}{j}\right|\right)<\phi(j)$, then this upper bound is an improvement on our original upper bound. If we repeat this process, this time simultaneously excluding the sets $S_{j}$ for all of the $j$ 's which gave us an improved upper bound in the above argument, we find that we have proved: 


\section{Proposition 2.1.}

$$
D(n) \leq \frac{n(n-1)}{2}+1-\sum_{j=1}^{n} \max \left(0, \phi(j)-D\left(n-j\left\lfloor\frac{n}{j}\right\rfloor\right)\right) .
$$

Finally, we obtain an upper bound for $D(n)$ which does not depend on other values of $D(\cdot)$ :

Corollary 2.2. $\quad$ Recursively define $U(0)=1$ and

$$
U(n)=\frac{n(n-1)}{2}+1-\sum_{j=1}^{n} \max \left(0, \phi(j)-U\left(n-j\left\lfloor\frac{n}{j}\right\rfloor\right)\right) .
$$

Then $D(n) \leq U(n)$.

Proof. We proceed by induction on $n$. Equality certainly holds for $n=0$. For larger $n$, the inductive hypothesis shows that $D\left(n-j\left\lfloor\frac{n}{j}\right\rfloor\right) \leq U\left(n-j\left\lfloor\frac{n}{j}\right\rfloor\right)$ when $j>0$, and so

$$
\begin{aligned}
D(n) & \leq \frac{n(n-1)}{2}+1-\sum_{j=1}^{n} \max \left(0, \phi(j)-D\left(n-j\left\lfloor\frac{n}{j}\right\rfloor\right)\right) \\
& \leq \frac{n(n-1)}{2}+1-\sum_{j=1}^{n} \max \left(0, \phi(j)-U\left(n-j\left\lfloor\frac{n}{j}\right\rfloor\right)\right) \\
& =U(n) .
\end{aligned}
$$

Below is a table of values of $D(n)$ and $U(n)$ for $1 \leq n \leq 34$, calculated for $1 \leq n \leq 23$ using Maple and for $24 \leq n \leq 34$ using a Python program. For contrast, $P(n)$ and our first estimate $\frac{n(n-1)}{2}+1$ are provided for $n \leq 24$, but are omitted (due to space considerations) for $n \geq 25$. Note that in the range $1 \leq n \leq 34$, we have $D(n)=U(n)$ except for $n=19,20,25,27,28,31$, when $U(n)-D(n)=1$, and $n=32,33$, when $U(n)-D(n)=2,3$ respectively. What is the behaviour of

$$
-D(n)+\frac{n(n-1)}{2}+1-\sum_{j=1}^{n} \max \left(0, \phi(j)-D\left(n-j\left\lfloor\frac{n}{j}\right\rfloor\right)\right)
$$

as $n \rightarrow \infty$ ?

Example 2.3. The first dimension where $D(n)<P(n)$ is $n=7$, and it is easy then to show that $D(n)<P(n)$ for all $n \geq 7$. The difference $P(7)-D(7)=2$ arises from the following two relations:

$$
\frac{4}{\left(1-x^{2}\right)^{2}(1-x)^{3}}=\frac{3}{\left(1-x^{3}\right)(1-x)^{4}}+\frac{1}{\left(1-x^{3}\right)\left(1-x^{2}\right)^{2}}
$$




\begin{tabular}{|c|r|r|r|r|r|r|r|r|r|r|r|r|r|r|}
\hline$n$ & 1 & 2 & 3 & 4 & 5 & 6 & 7 & 8 & 9 & 10 & 11 & 12 & 13 & 14 \\
\hline$D(n)$ & 1 & 2 & 3 & 5 & 7 & 11 & 13 & 19 & 23 & 29 & 35 & 45 & 51 & 62 \\
$U(n)$ & 1 & 2 & 3 & 5 & 7 & 11 & 13 & 19 & 23 & 29 & 35 & 45 & 51 & 62 \\
\hline$n(n-1) / 2+1$ & 1 & 2 & 4 & 7 & 11 & 16 & 22 & 29 & 37 & 46 & 56 & 67 & 79 & 92 \\
$P(n)$ & 1 & 2 & 3 & 5 & 7 & 11 & 15 & 22 & 30 & 42 & 56 & 77 & 101 & 135 \\
\hline
\end{tabular}

\begin{tabular}{|c|r|r|r|r|r|r|r|r|r|r|}
\hline$n$ & 15 & 16 & 17 & 18 & 19 & 20 & 21 & 22 & 23 & 24 \\
\hline$D(n)$ & 69 & 79 & 90 & 106 & 118 & 134 & 146 & 161 & 176 & 195 \\
$U(n)$ & 69 & 79 & 90 & 106 & 119 & 135 & 146 & 161 & 176 & 195 \\
\hline$n(n-1) / 2+1$ & 106 & 121 & 137 & 154 & 172 & 191 & 211 & 232 & 276 & 300 \\
$P(n)$ & 176 & 231 & 297 & 385 & 490 & 627 & 792 & 1002 & 1255 & 1575 \\
\hline
\end{tabular}

\begin{tabular}{|c|r|r|r|r|r|r|r|r|r|r|}
\hline$n$ & 25 & 26 & 27 & 28 & 29 & 30 & 31 & 32 & 33 & 34 \\
\hline$D(n)$ & 212 & 233 & 255 & 277 & 293 & 315 & 337 & 370 & 395 & 421 \\
$U(n)$ & 213 & 233 & 256 & 278 & 293 & 315 & 338 & 372 & 398 & 421 \\
\hline
\end{tabular}

Table 1: Values of $D(n), U(n), n(n-1) / 2+1, P(n)$ for small $n$

and

$$
\frac{3}{\left(1-x^{3}\right)\left(1-x^{2}\right)(1-x)^{2}}=\frac{2}{\left(1-x^{4}\right)(1-x)^{3}}+\frac{1}{\left(1-x^{4}\right)\left(1-x^{3}\right)} .
$$

The first relation, for example, says that if $\chi$ is a linear combination of $\chi_{N}$ 's, then

$$
4 \cdot \chi((2,2) \text {-cycle })=3 \cdot \chi(3 \text {-cycle })+\chi((3,2,2) \text {-cycle }) \text {. }
$$

Alternately, it tells us that for any $N \geq 0$, four times the number of nonnegative integral solutions to $2 x_{1}+2 x_{2}+x_{3}+x_{4}+x_{5}=N$ is equal to three times the number of such solutions to $3 x_{1}+x_{2}+x_{3}+x_{4}+x_{5}=N$ plus the number of such solutions to $3 x_{1}+2 x_{2}+2 x_{3}=N$.

\section{Lower Bounds on $D(n)$}

Let $\lambda=\left(\lambda_{1}, \ldots, \lambda_{k}\right) \vdash n$. The rational function $f_{\lambda}(q)$ of equation (1) can be written as

$$
f_{\lambda}(q)=p_{\lambda}\left(1, q, q^{2}, \ldots\right),
$$

where $p_{\lambda}$ denotes a power sum symmetric function. (See [1, Ch. 7] for the necessary background on symmetric functions.) Since the $p_{\lambda}$ for $\lambda \vdash n$ form a basis for the vector space (say over $\mathbb{C}$ ) $\Lambda^{n}$ of all homogeneous symmetric functions of degree $n[1$, Cor. 7.7.2], it follows that if $\left\{u_{\lambda}\right\}_{\lambda \vdash n}$ is any basis for $\Lambda^{n}$ then

$$
D(n)=\operatorname{dim} \operatorname{span}_{\mathbb{C}}\left\{u_{\lambda}\left(1, q, q^{2}, \ldots\right): \lambda \vdash n\right\} .
$$


In particular, let $u_{\lambda}=e_{\lambda}$, the elementary symmetric function indexed by $\lambda$. Define

$$
d(\lambda)=\sum_{i}\left(\begin{array}{c}
\lambda_{i} \\
2
\end{array}\right)
$$

According to [1, Prop. 7.8.3], we have

$$
e_{\lambda}\left(1, q, q^{2}, \ldots\right)=\frac{q^{d(\lambda)}}{\prod_{i}(1-q)\left(1-q^{2}\right) \cdots\left(1-q^{\lambda_{i}}\right)} .
$$

Since power series of different degrees (where the degree of a power series is the exponent of its first nonzero term) are linearly independent, we obtain from Proposition 1.2 the following result.

Proposition 3.1. Let $E(n)$ denote the number of distinct integers $d(\lambda)$, where $\lambda$ ranges over all partitions of $n$. Then $D(n) \geq E(n)$.

Note. We could also use the basis $s_{\lambda}$ of Schur functions instead of $e_{\lambda}$, since by $[1$, Cor. 7.21.3] the degree of the power series $s_{\lambda}\left(1, q, q^{2}, \ldots\right)$ is $d\left(\lambda^{\prime}\right)$, where $\lambda^{\prime}$ denotes the conjugate partition to $\lambda$.

Define $G(n)+1$ to be the least positive integer that cannot be written in the form $\sum_{i}\left(\begin{array}{c}\lambda_{i} \\ 2\end{array}\right)$, where $\lambda \vdash n$. Thus all integers $1,2, \ldots, G(n)$ can be so represented, so $D(n) \geq E(n) \geq G(n)$. We can obtain a relatively tractable lower bound for $G(n)$, as follows. For a positive integer $m$, write (uniquely)

$$
m=\left(\begin{array}{c}
k_{1} \\
2
\end{array}\right)+\left(\begin{array}{c}
k_{2} \\
2
\end{array}\right)+\cdots+\left(\begin{array}{c}
k_{r} \\
2
\end{array}\right),
$$

where $k_{1} \geq k_{2} \geq \cdots \geq k_{r} \geq 2$ and $k_{1}, k_{2}, \ldots$ are chosen successively as large as possible so that

$$
m-\left(\begin{array}{c}
k_{1} \\
2
\end{array}\right)-\left(\begin{array}{c}
k_{2} \\
2
\end{array}\right)-\cdots-\left(\begin{array}{c}
k_{i} \\
2
\end{array}\right) \geq 0
$$

for all $1 \leq i \leq r$. For instance, $26=\left(\begin{array}{l}7 \\ 2\end{array}\right)+\left(\begin{array}{l}3 \\ 2\end{array}\right)+\left(\begin{array}{l}2 \\ 2\end{array}\right)+\left(\begin{array}{l}2 \\ 2\end{array}\right)$. Define $\nu(m)=k_{1}+$ $k_{2}+\cdots+k_{r}$. Suppose that $\nu(m) \leq n$ for all $m \leq N$. Then if $m \leq N$ we can write $m=\left(\begin{array}{c}k_{1} \\ 2\end{array}\right)+\cdots+\left(\begin{array}{c}k_{r} \\ 2\end{array}\right)$ so that $k_{1}+\cdots+k_{r} \leq n$. Hence if $\lambda=\left(k_{1}, \ldots, k_{r}, 1^{n-\sum k_{i}}\right)$ (where $1^{s}$ denotes $s$ parts equal to 1 ), then $\lambda$ is a partition of $n$ for which $\sum_{i}\left(\begin{array}{c}\lambda_{i} \\ 2\end{array}\right)=m$. It follows that if $\nu(m) \leq n$ for all $m \leq N$ then $G(n) \geq N$. Hence if we define $H(n)$ to be the largest integer $N$ for which $\nu(m) \leq n$ whenever $m \leq N$, then we have established the string of inequalities

$$
D(n) \geq E(n) \geq G(n) \geq H(n) .
$$

Here is a table of values of these numbers for $1 \leq n \leq 23$. Note that $D(n)$ appears to be close to $E(n+1)$. We don't have any theoretical explanation of this observation. 


\begin{tabular}{|c|c|c|c|c|c|r|r|r|r|r|r|r|r|r|}
\hline$n$ & 1 & 2 & 3 & 4 & 5 & 6 & 7 & 8 & 9 & 10 & 11 & 12 & 13 & 14 \\
\hline$D(n)$ & 1 & 2 & 3 & 5 & 7 & 11 & 13 & 19 & 23 & 29 & 35 & 45 & 51 & 62 \\
$E(n)$ & 1 & 2 & 3 & 5 & 7 & 9 & 13 & 18 & 21 & 27 & 34 & 39 & 46 & 54 \\
$G(n)$ & 0 & 1 & 1 & 3 & 4 & 4 & 7 & 13 & 13 & 18 & 25 & 32 & 32 & 32 \\
$H(n)$ & 0 & 1 & 1 & 3 & 4 & 4 & 7 & 11 & 13 & 18 & 19 & 19 & 25 & 32 \\
\hline
\end{tabular}

\begin{tabular}{|c|r|r|r|r|r|r|r|r|r|}
\hline$n$ & 15 & 16 & 17 & 18 & 19 & 20 & 21 & 22 & 23 \\
\hline$D(n)$ & 69 & 79 & 90 & 106 & 118 & 134 & 146 & 161 & 176 \\
$E(n)$ & 61 & 72 & 83 & 92 & 106 & 118 & 130 & 145 & 162 \\
$G(n)$ & 40 & 49 & 52 & 62 & 73 & 85 & 102 & 112 & 127 \\
$H(n)$ & 40 & 43 & 52 & 62 & 73 & 85 & 89 & 102 & 116 \\
\hline
\end{tabular}

Table 2: Values of $D(n), E(n), G(n), H(n)$ for small $n$

Proposition 3.2. We have

$$
\nu(m) \leq \sqrt{2 m}+3 m^{1 / 4}
$$

for all $m \geq 405$.

Proof. The proof is by induction on $m$. It can be checked with a computer that equation (5) is true for $405 \leq m \leq 50000$. Now assume that $M>50000$ and that (5) holds for $405 \leq m<M$. Let $p=p_{M}$ be the unique positive integer satisfying

$$
\left(\begin{array}{l}
p \\
2
\end{array}\right) \leq M<\left(\begin{array}{c}
p+1 \\
2
\end{array}\right) .
$$

Thus $p$ is just the integer $k_{1}$ of equation (3). Explicitly we have

$$
p_{M}=\left\lfloor\frac{1+\sqrt{8 M+1}}{2}\right\rfloor \text {. }
$$

By the definition of $\nu(M)$ we have

$$
\nu(M)=p_{M}+\nu\left(M-\left(\begin{array}{c}
p_{M} \\
2
\end{array}\right)\right) .
$$

It can be checked that the maximum value of $\nu(m)$ for $m<405$ is $\nu(404)=42$. Set $q_{M}=(1+\sqrt{8 M+1}) / 2$. Since $M-\left(\begin{array}{c}p_{M} \\ 2\end{array}\right) \leq p_{M} \leq q_{M}$, by the induction hypothesis we have

$$
\nu(M) \leq q_{M}+\max \left(42, \sqrt{2 q_{M}}+3 q_{M}^{1 / 4}\right) .
$$

It is routine to check that when $M>50000$ the right hand side is less than $\sqrt{2 M}+$ $3 M^{1 / 4}$, and the proof follows. 
Proposition 3.3. There exists a constant $c>0$ such that

$$
H(n) \geq \frac{n^{2}}{2}-c n^{3 / 2}
$$

for all $n \geq 1$.

Proof. From the definition of $H(n)$ and Proposition 3.2 (and the fact that the right-hand side of equation (5) is increasing), along with the inquality $\nu(m) \leq 42=$ $\left\lceil\sqrt{2 \cdot 405}+3 \cdot 405^{1 / 4}\right\rceil$ for $m \leq 404$, it follows that

$$
H\left(\left\lceil\sqrt{2 m}+3 m^{1 / 4}\right\rceil\right) \geq m
$$

for $m>404$. For $n$ sufficiently large, we can evidently choose $m$ such that $n=$ $\left\lceil\sqrt{2 m}+3 m^{1 / 4}\right\rceil$, so $H(n) \geq m$. Since $\sqrt{2 m}+3 m^{1 / 4}+1>n$, an application of the quadratic formula (again for $n$ sufficiently large) shows

$$
m^{1 / 4} \geq \frac{-3+\sqrt{9+4 \sqrt{2}(n-1)}}{2 \sqrt{2}},
$$

from which the result follows without difficulty.

Since we have established both upper bounds (equation (2)) and lower bounds (equation (4) and Proposition 3.3) for $D(n)$ asymptotic to $n^{2} / 2$, we obtain the following corollary.

Corollary 3.4. There holds the asymptotic formula $D(n) \sim \frac{1}{2} n^{2}$.

\section{Acknowledgements}

The first author thanks Mark Dickinson for his help in computing values of $D(n)$.

\section{References}

[1] R. Stanley, Enumerative Combinatorics, vol. 2, Cambridge University Press, New York/Cambridge, 1999. 